\title{
Aorto-Right Atrial Fistula after Minimally Invasive Tricuspid Valve Repair: A Report of Two Cases
}

\author{
Kouhei Ishidou ${ }^{1}$ Aina Hirofuji ${ }^{1}$ Naohiro Wakabayashi ${ }^{1}$ Hiroyuki Kamiya ${ }^{1}$ \\ ${ }^{1}$ Department of Cardiac Surgery, Asahikawa Medical University, \\ Asahikawa, Japan \\ ${ }^{2}$ Asahikawa Medical University, Asahikawa, Hokkaido, Japan \\ Address for correspondence Hiroyuki Kamiya, MD, PhD, Department \\ of Cardiac Surgery, Asahikawa Medical University, Midorigaoka \\ Higashi 2-1-1-1, Asahikawa 078-8510, Japan (e-mail:
}

Thorac Cardiovasc Surg Rep 2020;9:e55-e57.

\begin{abstract}
Keywords

- minimally invasive cardiac surgery

- aorto-right atrial fistula

- tricuspid valve repair

We experienced two cases of postoperative iatrogenic aorto-right atrial fistula (ARAF) after tricuspid valve repair (TVR) using minimally invasive cardiac surgery (MICS) technique. In both the cases, the flow of ARAF passed through the sinus of Valsalva near the noncoronary cusp (NCC)/right coronary cusp (RCC) commissure or NCC to right atrium. The quality of the fine needle used in the MICS technique may be inferior to that used in conventional surgery; ARAF after TVR could be a unique pitfall with the MICS technique.
\end{abstract}

\section{Introduction}

Aorto-right atrial fistula (ARAF) after tricuspid valve repair (TVR) is a rare complication. We experienced two cases of postoperative iatrogenic ARAF after TVR using the minimally invasive cardiac surgery (MICS) technique. Herein, we report these two cases.

\section{Case Reports}

\section{Case 1}

An 86-year-old man with longstanding congestive heart failure due to chronic atrial fibrillation (cAf) was referred to our department due to acute worsening of dyspnea. A transthoracic echocardiogram (TTE) showed severe mitral regurgitation (MR) and severe tricuspid regurgitation (TR); both MR and TR were resultant of massive dilatation of the annulus due to cAf. Mitral valve repair (MVR) with implantation of annuloplasty ring (IMR ETlogix $32 \mathrm{~mm}$, Edwards Lifesciences, Irvine, California, United States) and TVR with implantation of annuloplasty ring (Physio Tricuspid $30 \mathrm{~mm}$, Edwards Lifesciences) were performed via right minithoracotomy as an MICS. In this case, TVR was performed during cardioplegic arrest. Postoperative TTE showed mild MR and TR. The postoperative course was uneventful and the patient was transferred to a different hospital for rehabilitation on postoperative day (POD) 13.
A follow-up TTE after 9 months revealed ARAF, which was not present at the postoperative TTE. The shunt jet passed through the sinus of Valsalva near the noncoronary cusp (NCC)/ right coronary cusp (RCC) commissure to the anterior annulus of the tricuspid valve near the commissure between the septal leaflet and the anterior leaflet ( $\mathbf{F i g . 1}$ ). Pulmonary blood flow ratio could not be measured because the shunt volume was trivial. Owing to the small value of the shunt flow, the extremely high age and no remarkable symptoms, the patient was henceforth managed conservatively.

\section{Case 2}

A 90-year-old woman with a similar history as the case 1 underwent MVR with implantation of annuloplasty ring (Physio I $30 \mathrm{~mm}$, Edwards Lifesciences) and TVR with implantation of annuloplasty ring (Physio Tricuspid $26 \mathrm{~mm}$, Edwards Lifesciences) via a right minithoracotomy using the MICS technique in the completely same fashion as the case 1 . The postoperative course was uneventful. A postoperative TTE showed no residual MR and mild TR; however, an ARAF was detected which was not present in the intraoperative transesophageal echocardiogram (-Fig. 2). Similar to case 1 , the shunt flow passed through the sinus of Valsalva near the NCC to the right atrium and the shunt volume was minimal. The patient was discharged on POD 16 and has been conservatively managed as well.
License terms

Stuttgart · New York

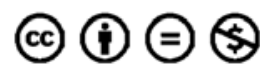

July 7, 2020

accepted after revision

September 7, 2020
DOI https://doi.org/

10.1055/s-0040-1718552. ISSN 2194-7635. 


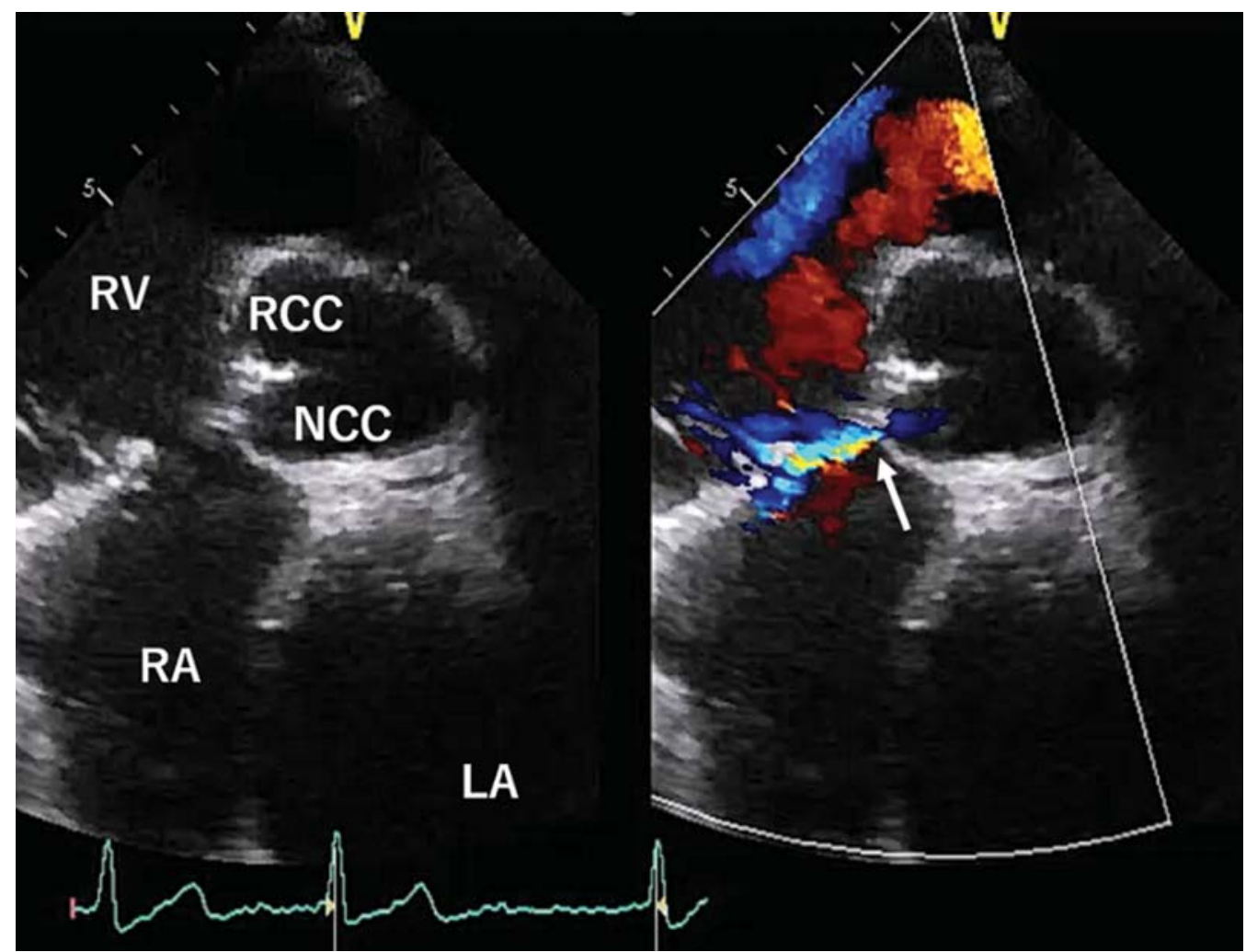

Fig. 1 TTE of ARAF. ARAF flow through the sinus of Valsalva near the RCC-NCC commissure to the tricuspid annuloplasty ring and the other side of the tricuspid annulus as shown by TTE with Doppler. ARAF, aorto-right atrial fistula; LA, left atrium; NCC, noncoronary cusp; RA, right atrium; RCC, right coronary cusp; RV, right ventricle; TTE, transthoracic echocardiogram.

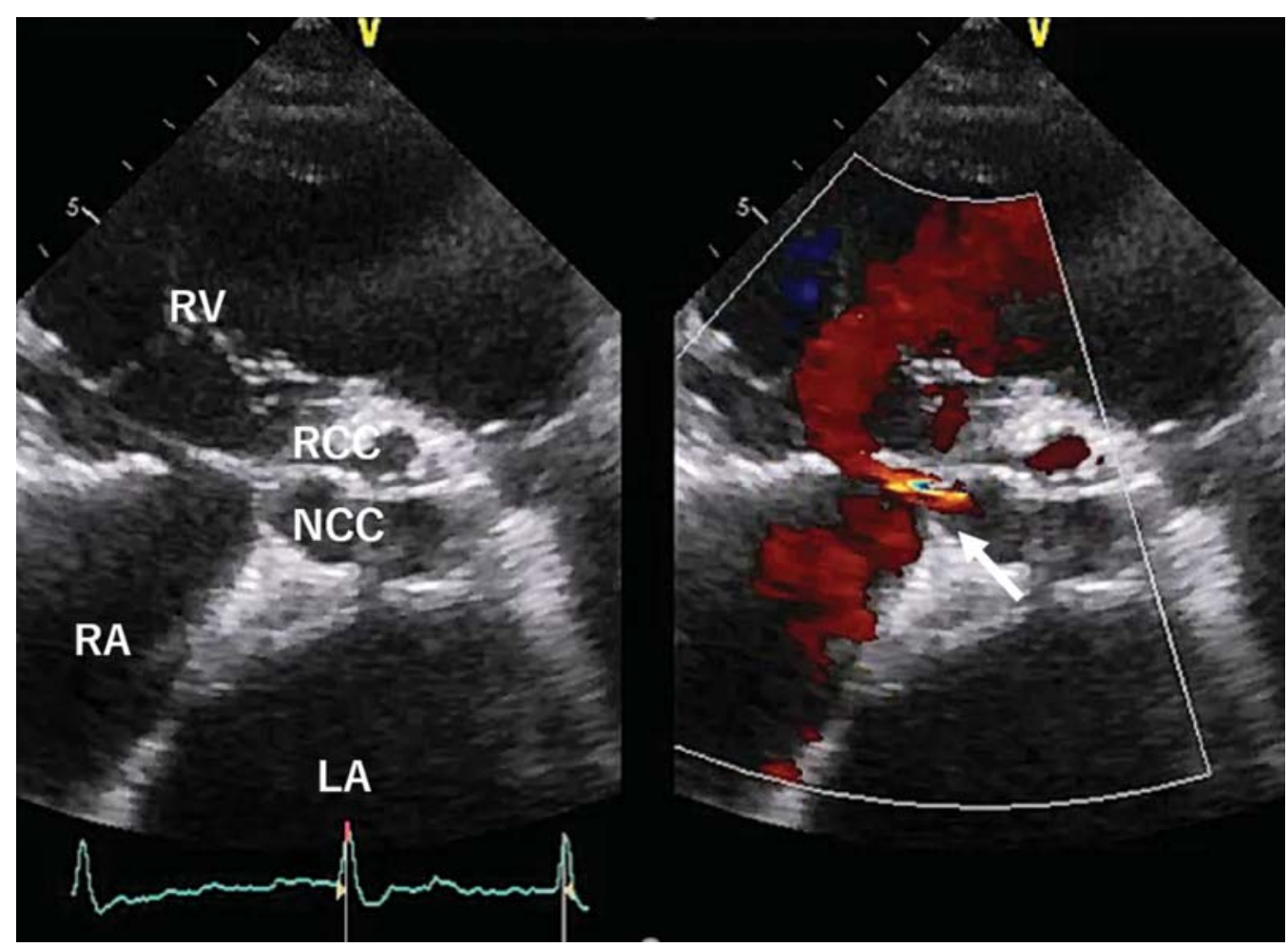

Fig. 2 TTE of ARAF. ARAF flow through the sinus of Valsalva near the NCC to the right atrium as shown by TTE with Doppler. ARAF, aorto-right atrial fistula; LA, left atrium; NCC, noncoronary cusp; RA, right atrium; RCC, right coronary cusp; RV, right ventricle; TTE, transthoracic echocardiogram. 


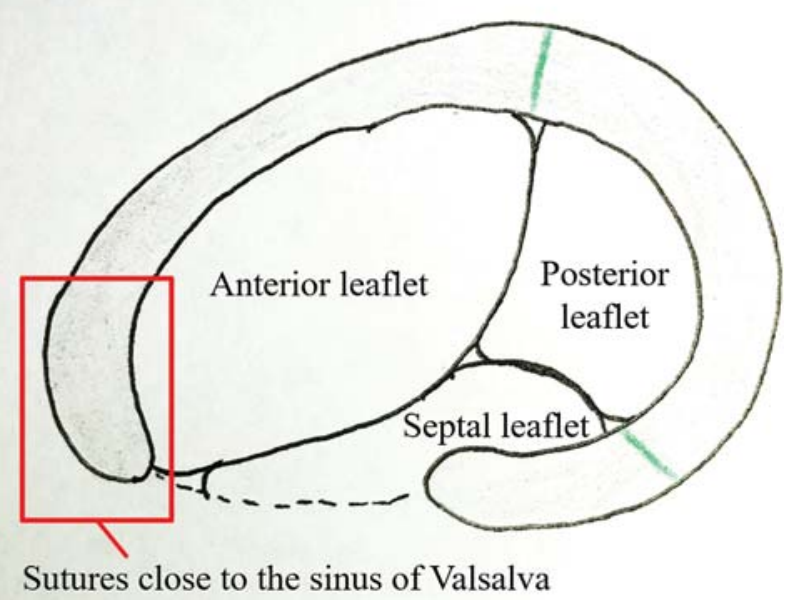

Fig. 3 Sutures at the anterior annulus close to the sinus of Valsalva for anchoring the annuloplasty ring.

\section{Discussion}

We experienced two cases of iatrogenic ARAF after minimally invasive TVR. To the best of our knowledge, these cases have shown the first description of ARAF as a complication of minimally invasive TVR. In both our cases, the flow of ARAF passed through the sinus of Valsalva near the NCC-RCC commissure or the NCC to the right atrium.

It was reported that aorto-cardiac fistula occurred with infective endocarditis, blunt trauma, stab wound to the chest, ruptured aneurysms of the sinus of Valsalva, aortic dissection, congenital disorder, cardiac valve surgery, percutaneous cardiac valve implantation, heart transplantation, and autoimmune vasculitis. ${ }^{1}$ In our cases, annular debridement was not performed, and there were no symptoms of infective endocarditis near the tricuspid valve. In addition, ARAF showed only in postoperative examination. Therefore, we believe that ARAFs in the two presented cases were iatrogenic.

Aoyagi et al reported a case of left ventricular-right atrial communication after TVR. The TVR ring could probably have been placed on the atrioventricular portion of the membranous septum, rather than the tricuspid annulus, at the anteroseptal commissure of the tricuspid valve and its dehiscence may have created a tear in the atrioventricular membranous septum, leading to left ventricular-right atrial communication. ${ }^{2}$ In our cases, considering that ARAF flow was through the sinus of Valsalva near the NCC-RCC commissure or the NCC to the right atrium, we speculate that the sutures for anchoring the annuloplasty ring at the anterior annulus of the tricuspid valve (-Fig. 3) may have caused ARAF.

In addition, the MICS technique may affect the occurrence of ARAF as it is very uncommon after conventional TVR through the median sternotomy. In the MICS technique, the working space is smaller and deeper than in the sternotomy approach, and therefore, long shaft instruments are needed. It might be possible that the quality of the fine needle used in the MICS technique may have been inferior to that in conventional surgery, and one should be aware that ARAF after TVR could be a unique pitfall with the MICS technique.

Recently, a novel prosthesis for TVR (Tri-Ad, Medtronic, Minneapolis, Minnesota, United States) has been developed in which suturing at the anterior annulus close to the sinus of Valsalva is not necessary. From our experience, we consider that ARAF in minimally invasive TVR might be avoided by using this novel prosthesis. ${ }^{3}$

\section{Conclusion}

In conclusion, we reported two cases of iatrogenic ARAF after minimally invasive TVR. One should be aware that ARAF after TVR might be a unique pitfall with the MICS technique.

Conflict of Interest

None declared.

\section{References}

1 Villablanca PA, Sukhal S, Maitas O, et al. Aorto-right atrial fistula: late complication of tricuspid valve infective endocarditis. World J Cardiol 2014;6(10):1122-1126

2 Aoyagi S, Arinaga K, Oda T, Hori H. Left ventricular-right atrial communication following tricuspid annuloplasty. Eur J Cardiothorac Surg 2008;34(03):680-681

3 Jainandunsing JS, Linnemann R, Bouma W, et al. Aorto-atrial fistula formation and closure: a systematic review. J Thorac Dis 2019;11(03):1031-1046 